\title{
Local and global asymptotic behavior of malaria-filariasis coinfections in compliant and noncompliant susceptible pregnant women to antenatal medical program in the tropics
}

\author{
Oluwatayo M. Ogunmiloro \\ Communicated by Valery Covachev
}

\begin{abstract}
In this paper, a mathematical nonlinear model system of equations describing the dynamics of the co-interaction between malaria and filariasis epidemic affecting the susceptible host population of pregnant women in the tropics is formulated. The basic reproduction number $R_{m f}$ of the coepidemic model is obtained, and we investigated that it is the threshold parameter between the extinction and persistence of the coepidemic disease. If $R_{m f}<1$, then the disease-free steady state is both locally and globally asymptotically stable resulting in the disease dying out of the host. Also, if $R_{m f}>1$, the disease lingers on. The center manifold theory is used to show that the unique endemic equilibrium is locally asymptotically stable. However, variations in the parameter values involved in the model build up will bring about appropriate control measures to curtail the spread of the coepidemic disease. Numerical simulations are carried out to confirm the theoretical results.
\end{abstract}

Keywords. Malaria-filariasis, coinfection, reproduction number, center manifold, local stability.

2010 Mathematics Subject Classification. 92B05, 93D05, 34K12, 34K20.

\section{Introduction}

Coinfection is an infection of a single host simultaneously by a causative agent with multiple pathogens which possess various strains of the disease. Malaria filariasis coinfection is an old parasitic disease that still poses a serious threat and a medical challenge to the global world population, where the endemic impact is most felt in the developing and third world countries in Africa and Asia. These two coendemic diseases account for the world largest mortality and morbidity respectively. According to the World Health Organization (WHO) fact sheet of 2018 [1], malaria killed an estimated 438, 000 individuals, where over 90 percent deaths occurred in Africa, while 10 and 2 percents deaths were recorded in Asia and the 
Eastern Mediterranean Region respectively. Malaria is a deadly parasitic disease transmitted to the human body through the bites of infected mosquitos, mainly female Anopheles mosquito. The clinical signs associated with malaria after few days are tiredness, headache, high fever, chills, etc.

Lymphatic filariasis is another debilitating mosquito-borne disease caused by parasitic nematode of filaroidea family. There are three types of filaria parasites, namely, Wuchereria bancrofti, which is responsible for 90 percent of cases, Brugia timori, Brugia malayi. According to WHO fact sheet of 2018 [2], 856 million people in 52 countries remain threatened by filariasis and require preventive chemotherapy to curtail the spread of this parasitic disease. Infection happens when filarial parasites are deposited in human body through mosquito bite, thereby causing damage to the lymphatic system. The filaria worms can live in the lymphatic vessels for $6-8$ years, producing millions of microfilariae (immature larvae) that circulate in the blood. Infected humans exhibit clinical manifestation of lymphoedema, elephantiasis, body deformation, thereby leading to social stigma. Nigeria is one of the subtropical countries where this codisease thrives. A. G. Abdullahi et al. [8], reported the presence of lymphatic filariasis and malaria in rural communities in Nassarawa state of Nigeria, see also [3,13]. In 2014, Nigeria launches Africa's first nationwide malaria and lymphatic filariasis elimination coimplementation plan to protect the citizens from this dreaded disease $[5,7]$.

In this paper, our focus is on analyzing the dynamics of the spread of coinfectious malaria - filariasis disease among susceptible pregnant women in Nigeria, who are compliant or noncompliant to antenatal medical program to access treatment and care to reduce cases of maternal and foetal mortality $[4,6,9]$. The works of $[10-12,14-22,24]$ have proved very useful in the understanding of coinfection dynamics of the spread of diseases. Having gone through all the cited literatures above, and motivated by the works of H.C. Slater [23] and [12, 25 - 29], in this study, a mathematical coendemic deterministic model describing the qualitative dynamics of malaria - filariasis coinfections considering pregnant women only is considered. The rest of the paper is organized as follows: Section 2 presents the analytical discussion and formulation of malaria - filariasis coinfection transmission model with corresponding initial conditions. In Section 3, the basic reproduction number $R_{m f}$ is obtained. Also, in Section 4, the time-independent steady-state solutions are found where the malaria - filariasis coinfection is present and absent in the host population, while the necessary and sufficient conditions for the asymptotic stability of the coinfections at disease-free steady state are investigated both locally and globally. Furthermore, the endemic steady state is analyzed by employing the center manifold theory. Section 5 deals with the numerical simulations and graphical illustrations of the behavior of the variables and parameters involved in the model. Also, conclusions and recommendations were made. 


\section{Mathematical model formulation}

In this model, the total pregnant women population at time $t$ denoted $N_{h}$ is subdivided into disjoint sub-populations of individual pregnant women who are compliant to medical antenatal program denoted $S_{h}$; Individual susceptible women who are noncompliant to medical antenatal program as $S_{2}$; Individual pregnant women infected with malaria only $I_{m}$; Individual pregnant women infected with filariasis only $I_{f}$; Individual pregnant women infected with both malaria and filariasis $I_{m f}$; Individual pregnant women treated from malaria - filariasis coinfections as $T$; Thus, $N_{h}=S_{h}+S_{2}+I_{m}+I_{f}+I_{m f}+T$. Since the spread of malaria - filariasis is being carried out by the same vector (Anopheles mosquito), the total vector population is denoted $N_{v}$; Susceptible mosquito as $S_{v}$; and exposed mosquito as $E_{v}$, and infected mosquito as $I_{v}$, such that $N_{v}=S_{v}+E_{v}+I_{v}$.

The population of the susceptible pregnant women compliant to medical antenatal program is recruited into the host population at constant per capita rate $A_{h}$, while $\pi$ and $\tau$ are represented as the fraction of susceptible pregnant women that are compliant to antenatal medical program, and loss of immunity to the codisease respectively. This group is further reduced by natural death rate $\mu_{h}$ and $\delta$ as the progression rate from $S_{h}$ to $S_{2}$ group and force of infection $K_{m a} S_{h}$ and $K_{f} S_{h}$ respectively. Hence the rate of change of the host population is thus given as

$$
\dot{S}_{h}=\pi A_{h}-\left(K_{m a}+K_{f}\right) S_{h}-\left(\mu_{h}+\delta\right) S_{h}+\tau T .
$$

Also, $S_{2}$ is generated by $(1-\pi) A_{h}$ individual susceptible pregnant women who are noncompliant to antenatal medical program, progression rate $\delta_{1}$, and force of infection $\left(K_{m a}+K_{f}\right) S_{h}$. It is reduced by mortality rate due to $S_{2}$ group, natural death rate $\mu_{h}$, and $\epsilon \sigma_{h}$, where $\epsilon$ is the modification parameter representing the assumption that filariasis infected individuals exposed to malaria develop malaria faster than those infected with filariasis. Also, $\sigma_{h}$ is the rate of malaria symptoms by susceptible pregnant women noncompliant to antenatal medical program, while $\gamma$ is the disease-induced death rate. Thus, the rate of change is given by

$$
\dot{S}_{2}=(1-\pi) A_{h}+\delta_{1} S_{h}+\left(K_{m a}+K_{f}\right) S_{h}-\left(\epsilon \sigma_{h}+\gamma+\mu_{h}\right) S_{2} .
$$

Furthermore, the rate of change of $I_{m}$ class is increased by $\epsilon \sigma_{h} S_{2}$ and decreased by $\theta K_{f} I_{m}$, where $\theta$ takes into account the increased susceptibility to infection with filariasis for individuals infected with malaria. Also, it is further reduced by the quantity $\left(\psi+\phi_{1}+\mu_{h}\right) I_{m}$, where $\phi_{1}, \mu_{h}, \psi$ are the treatment rate, natural death rate, and progression rate respectively. Hence, it is given by 


$$
\dot{I_{m}}=\epsilon \sigma_{h} S_{2}-\theta K_{f} I_{m}-\left(\psi+\phi_{1}+\mu_{h}\right) I_{m} .
$$

Also, $I_{f}$ class is increased with progression rate $\psi I_{m}$ but decreased by $\rho K_{m a} I_{f}$, where $\rho$ takes into account the decreased susceptibility to infection with malaria for individuals infected with filariasis, $\phi_{2} I_{f}$ as the recovery rate from filariasis through treatment, natural death rate $\mu_{h} I_{f}$. Thus, the state equation is given by

$$
\dot{I_{f}}=\psi I_{m}-\rho K_{m a} I_{f}-\left(\gamma+\phi_{2}+\mu_{h}\right) I_{f}
$$

The rate of change of population of individual pregnant women infected with both malaria and filariasis is increased by $\theta K_{f} I_{m}$ and $\rho K_{m a} I_{f}$ but decreased by recovery rate $\phi_{3} I_{m f}, v \phi I_{m f}$ as the treatment rate for the coinfection, $\eta \gamma I_{m f}$ is the codisease induced death rate and natural death rate $\mu_{h} I_{m f}$. Hence, it is given by

$$
I_{m f}^{\cdot}=\theta K_{f} I_{m}+\rho K_{m a} I_{f}-\left(\phi_{3}+v \phi+\eta \gamma+\mu_{h}\right) I_{m f} .
$$

The rate of change of population of treated pregnant women infected with both malaria and filariasis is increased by $v \phi I_{m f}, \phi_{1} I_{m}, \phi_{2} I_{f}, \phi_{3} I_{m f}$ but decreased by natural death rate $\mu_{h} T$ and loss of immunity rate $\tau T$. Then

$$
\dot{T}=v \phi I_{m f}+\left(\phi_{1} I_{m}+\phi_{2} I_{f}+\phi_{3} I_{m f}\right)-\left(\mu_{h}+\tau\right) T .
$$

The rate of change of population of susceptible mosquito is generated by the recruitment of mosquitos $A_{v}$ and decreased by the natural death rate $\mu_{v} S_{v}$ and contact rate $K_{v} S_{v}$ between susceptible and exposed class such that

$$
\dot{S}_{v}=A_{v}-K_{v} S_{v}-\mu_{v} S_{v}
$$

The rate of change of population of exposed mosquito is generated by the force of infection $K_{v}$ and decreased by progression rate of mosquito $\sigma_{v}$ and natural death rate of mosquito $\mu_{v}$. Thus, it is given by

$$
\dot{E}_{v}=K_{v} S_{v}-\sigma_{v} E_{v}-\mu_{v} E_{v}
$$

Finally, the rate of change of population of infected mosquito class $I_{v}$ is generated by

$$
I_{v}=\sigma_{v} E_{v}-\mu_{v} I_{v}
$$

The notation

$$
K_{m a}=\frac{\alpha a I_{v}}{N_{h}}
$$


where $\alpha$ is the biting rate of mosquitos and $a$ is the transmission probability of malaria in pregnant women per bite, is introduced. Also

$$
K_{f}=\frac{\beta\left(I_{m}+I_{m f}+k I_{f}\right)}{N_{h}},
$$

where $b$ is the probability of transmission in mosquitos from any infected pregnant women, $\beta$ is the effective contact rate of infection between the infective classes, while

$$
K_{v}=\frac{\alpha b\left(I_{m}+\delta I_{m f}\right)}{N_{h}} .
$$

Here, $\delta$ is the modification parameter that takes into account the increase in the likelihood of infection of mosquitos from susceptible pregnant women with coinfection of malaria - filariasis in relation to acquiring infection from pregnant women with malaria only. Also, $k$ is the modification parameter that accounts for the relative infectiousness of asymptomatically infected individual with filariasis exposed to malaria.

Assumptions in the model build-up are:

- The total population of pregnant women in the tropics is considered alone.

- There are birth and death rates.

- There is no vertical transmission between the mother and the child.

- Treatment is carried out for both pregnant women that are infected with malaria or filariasis.

- After treatment, immunity is lost, thereby making the pregnant women to be susceptible to the codisease again.

Hence the full malaria - filariasis model is given by

$$
\begin{aligned}
\dot{S}_{h} & =\pi A_{h}-K_{m a} S_{h}-K_{f} S_{h}-\left(\mu_{h}+\delta\right) S_{h}+\tau T, \\
\dot{S}_{2} & =(1-\pi) A_{h}+\delta S_{h}+K_{m a} S_{h}+K_{f} S_{h}-\left(\epsilon \sigma_{h}+\gamma+\mu_{h}\right) S_{2}, \\
\dot{I_{m}} & =\epsilon \sigma_{h} S_{2}-\theta K_{f} I_{m}-\left(\psi+\phi_{1}+\mu_{h}\right) I_{m}, \\
\dot{I_{f}} & =\psi I_{m}-\rho K_{m a} I_{f}-\left(\gamma+\phi_{2}+\mu_{h}\right) I_{f}, \\
I_{m f} & =\theta K_{f} I_{m}+\rho K_{m a} I_{f}-\left(\phi_{3}+v \phi+\eta \gamma+\mu_{h}\right) I_{m f}, \\
\dot{T} & =v \phi I_{m f}+\left(\phi_{1} I_{m}+\phi_{2} I_{f}+\phi_{3} I_{m f}\right)-\mu_{h} T-\tau T \\
\dot{S_{v}} & =A_{v}-K_{v} S_{v}-\mu_{v} S_{v} \\
\dot{E_{v}} & =K_{v} S_{v}-\left(\sigma_{v}+\mu_{v}\right) E_{v} \\
\dot{I_{v}} & =\sigma_{v} E_{v}-\mu_{v} I_{v}
\end{aligned}
$$


subject to initial conditions $S_{h}(0)=S_{h o}, S_{2}(0)=S_{2 o}, I_{m}(0)=I_{m o}, I_{f}(0)=$ $I_{f o}, I_{m f}(0)=I_{m f o}, T(0)=T_{o}, S_{v}(0)=S_{v o}, E_{v}(0)=E_{v o}, I_{v}(0)=I_{v o}$.

\subsection{Positivity and boundedness of solutions}

The solutions of the malaria - filariasis model with nonnegative initial data are nonnegative for all time $t>0[17,19]$.

Theorem 2.1. If $S_{h}(0), S_{2}(0), I_{m}(0), I_{f}(0), I_{m f}(0), T(0), S_{v}(0), E_{v}(0), I_{v}(0)$ are nonnegative, then $S_{h}(t), S_{2}(t), I_{m}(t), I_{f}(t), I_{m f}(t), T(t), S_{v}(t), E_{v}(t), I_{v}(t)$

are defined for all $t>0$, such that

$$
\lim _{t \rightarrow \infty} \sup N_{h}(t) \leq \frac{A_{h}}{\mu_{h}}
$$

and

$$
\lim _{t \rightarrow \infty} \sup N_{v}(t) \leq \frac{A_{v}}{\mu_{v}},
$$

which implies that

$$
N_{h}(0) \leq \frac{A_{h}}{\mu_{h}}, \quad N_{v}(0) \leq \frac{A_{v}}{\mu_{v}} .
$$

The feasible region for (13) is given by

$$
\xi=\left(\xi_{h}\right)\left(\xi_{v}\right) \subset \Re_{+}^{6} \times \Re_{+}^{3},
$$

where

$$
\begin{gathered}
\xi_{h}=\left[\left(S_{h}, S_{2}, I_{m}, I_{f}, I_{m} f, T\right) \in \Re_{+}^{6}:\right. \\
\left.S_{h}+S_{2}+I_{m}+I_{f}+I_{m f}+T \leq \frac{A_{h}}{\mu_{h}}\right]
\end{gathered}
$$

and

$$
\xi_{v}=\left[\left(S_{v}, E_{v}, I_{v}\right) \in \Re_{+}^{3}: S_{v}+E_{v}: \leq \frac{A_{v}}{\mu_{h}}\right]
$$

show that $\xi$ is a positively invariant region. 
Proof. Since

$$
S_{h}(0), S_{2}(0), I_{m}(0), I_{f}(0), I_{m f}(0), T(0), S_{v}(0), E_{v}(0), I_{v}(0)
$$

are nonnegative, where $t_{1}>0$, such that

$$
t_{1}=\sup \left[t>0: S_{h}, S_{2}, I_{m}, I_{f}, I_{m f}, T, S_{v}, E_{v}, I_{v} \text { are nonnegative on }[0, t]\right] .
$$

If $t_{1}<\infty$, from the first equation of (13), we have

$$
S_{h}\left(t_{1}\right)=Z\left(t_{1}, 0\right) S_{h}(0)+\int_{0}^{t_{1}} A \Omega\left(t_{1}, \varsigma\right) d \varsigma,
$$

where

$$
Z\left(t_{1}, \varsigma\right)=e^{-\int_{\varsigma}^{t}\left(K_{m a}+K_{f}+\mu_{h}+\delta\right)(s) d s} .
$$

Furthermore, the sum of the last three state equations in (13) is given by

$$
\dot{N}_{v}=A_{v}-\left(S_{v}+E_{v}+I_{v}\right) \mu_{v}=A_{v}-N_{v} \mu_{v}
$$

so that,

$$
N_{v}(t)=N_{v}(0) e^{-\mu_{v} t}+\frac{A_{v}}{\mu_{v}}\left(1-e^{-\mu_{v} t}\right)
$$

and

$$
\lim \sup _{t \rightarrow \infty} N_{v}(t)=\frac{A_{v}}{\mu_{v}}
$$

From (26), it follows that

$$
N_{v}(0) \leq \frac{A_{v}}{\mu_{v}}
$$

and

$$
N_{v}(0) \leq \frac{A_{v}}{\mu_{v}}
$$

Moreover, the addition of the first six state equations in (13) yields

$$
N_{h}=A_{h}-\left(S_{h}+S_{2}+I_{m}+I_{n}+I_{m} f+T\right) \mu_{h}-\gamma S_{2}-\gamma I_{f}-\eta \gamma I_{m f},
$$

so that, in the absence of disease-induced mortality, (29) becomes

$$
\dot{N}_{h} \leq A_{h}-\mu_{h} N_{h},
$$


then

$$
A_{h}-\left(\mu_{h}+\gamma+\eta\right) N_{h} \leq \dot{N}_{h} \leq A_{h}-\mu_{h} N_{h} .
$$

Applying the standard comparison theorem method yields

$$
N_{h}(0) e^{\left(\mu_{h}+\gamma+\eta t\right)}+\frac{A_{h}}{\mu_{h}+\gamma+\eta}\left(1-e^{\left(\mu_{h}+\gamma+\eta\right) t}\right) \leq N_{h} \leq N_{h}(0) e^{-\mu_{h} t},
$$

so that

$$
N_{h}(0) \leq \frac{A_{h}}{\mu_{h}}
$$

and

$$
N_{h}(t) \leq \frac{A_{h}}{\mu_{h}} .
$$

Thus, the malaria - filariasis model is epidemiologically feasible and mathematically well - posed.

\section{Basic reproduction number $\left(\boldsymbol{R}_{m f}\right)$}

The basic reproduction number $\left(R_{m f}\right)$ is a measure of potential for the malaria - filaria disease spread in a susceptible host population of pregnant women. If $R_{m f}<1$, few infectives introduced into the population will fail to replace themselves leading to the eradication of the disease. However, if $R_{m f}>1$, the total number of infectives will increase which in turn leads to disease spread and mortality until optimum strategies are considered in order to curtail the spread of the disease. Also, if $R_{m f}<1$, the disease-free equilibrium is locally asymptotically stable. There exists the endemic equilibrium if $R_{m f}>1$.

Theorem 3.1. [24] Let $x=\left(x_{1}, x_{2}, \ldots, x_{n}\right)^{T}$ be the number of individuals in each compartment, where the first $m<n$ compartments contain infected individuals.

$$
\frac{d x_{i}}{d t}=F_{i}(x)-V_{i}(x), \quad i=1,2, \ldots . m,
$$

where $f_{i}(x)$ is the rate of appearance of new infections in compartment $i . V_{i}(x)$ $i$ is the rate of either transition between compartment $i$ and other infected compartments, such that

$$
F=\left[\frac{\partial f_{1}\left(x_{o}\right)}{\partial x_{j}}\right], \quad V=\left[\frac{\partial V_{i}\left(x_{o}\right)}{\partial x_{j}}\right], \quad 1 \leq i, j \leq m,
$$


$F$ is entrywise nonnegative and $V$ is a nonsingular $M$-matrix. Also, $V^{-1}$ is entrywise nonnegative. Thus, the matrix $\left(F V^{-1}\right)$ has $[i, j]$ entry equal to the expected number of secondary infections in compartment $i$ produced by an infected individual introduced in compartment $j . F V^{-1}$ is the next generation matrix and $R_{0}=\rho\left(F V^{-1}\right)$, where $\rho$ is the spectral radius.

Proof. We use the next generation matrix method [24]. Suppose that

$$
\begin{aligned}
& F=\left(\begin{array}{ccccccccc}
0 & 0 & 0 & 0 & 0 & 0 & 0 & 0 & 0 \\
0 & 0 & \beta & \beta k & \beta & 0 & 0 & 0 & 0 \\
0 & 0 & 0 & 0 & 0 & 0 & 0 & 0 & 0 \\
0 & 0 & 0 & 0 & 0 & 0 & 0 & 0 & 0 \\
0 & 0 & \theta \beta & \theta \beta k & \theta \beta & 0 & 0 & 0 & 0 \\
0 & 0 & 0 & 0 & 0 & 0 & 0 & 0 & 0 \\
0 & 0 & 0 & 0 & 0 & 0 & 0 & 0 & 0 \\
0 & 0 & 0 & 0 & 0 & 0 & K_{v} & 0 & 0 \\
0 & 0 & 0 & 0 & 0 & 0 & 0 & 0 & 0
\end{array}\right), \\
& V=\left(\begin{array}{ccccccccc}
J_{1} & 0 & 0 & 0 & 0 & \tau & 0 & 0 & 0 \\
\delta & J_{2} & 0 & 0 & 0 & 0 & 0 & 0 & 0 \\
0 & \epsilon \sigma_{h} & J_{3} & 0 & 0 & 0 & 0 & 0 & 0 \\
0 & 0 & \psi & J_{4} & 0 & 0 & 0 & 0 & 0 \\
0 & 0 & 0 & 0 & J_{5} & 0 & 0 & 0 & 0 \\
0 & 0 & \phi_{1} & \phi_{2} & v \phi+\phi_{3} & J_{6} & 0 & 0 & 0 \\
0 & 0 & 0 & 0 & 0 & 0 & J_{7} & 0 & 0 \\
0 & 0 & 0 & 0 & 0 & 0 & 0 & J_{8} & 0 \\
0 & 0 & 0 & 0 & 0 & 0 & 0 & \sigma_{v} & J_{9}
\end{array}\right),
\end{aligned}
$$

where $J_{1}=\left(\mu_{h}+\delta\right), J_{2}=\left(\epsilon \sigma_{h}+\gamma+\mu_{h}\right), J_{3}=\left(\psi+\phi_{1}+\mu_{h}\right), J_{4}=\left(\gamma+\phi_{2}+\mu_{h}\right)$, $J_{5}=\left(\phi_{3}+v \phi+n \gamma+\mu_{h}\right), J_{6}=\left(\mu_{h}+\tau\right), J_{7}=\mu_{v}, J_{8}=\left(\sigma_{v}+\mu_{v}\right), J_{9}=\mu_{v}$,

$$
R_{m f}\left(F V^{-1}\right)=\frac{\epsilon \sigma_{h}\left(J_{6} J_{1}\left(\beta k \psi-\beta J_{4}\right)+\tau \delta\left(\psi \theta \beta k-\theta \beta J_{4}\right)\right)}{\tau \delta\left(\psi \phi_{2}-J_{4} \phi_{3}\right) \epsilon \sigma_{h}+J_{1} J_{2} J_{3} J_{4} J_{5} J_{6}} .
$$




\section{Stability analysis of the model}

\subsection{Local stability analysis of disease-free steady state}

Theorem 4.1. [25] The disease-free equilibrium is asymptotically stable if $R_{m f} \leq$ 1 and unstable if $R_{m f}>1$.

Proof. Firstly, we analyze the malaria - filaria codisease qualitatively to investigate the steady states of the model. We make the model system static by equating the right-hand side of the model to zero, that is, the system does not depend on time, such that

$$
\dot{S}_{h}=\dot{S}=\dot{I}_{m}=\dot{I}_{f}=I_{m f}=\dot{T}=0, \quad \dot{S}_{v}=\dot{E}_{v}=\dot{I}_{v}=0 \text {. }
$$

Therefore, the steady states are given by

$$
\begin{gathered}
\left(S_{h}=\frac{\pi A_{h}}{\mu_{h}}, S_{2}=\frac{A_{h}}{\mu_{h}}, I_{m}=0, I_{f}=0, I_{m f}=0, T=0,\right), \\
\left(S_{v}=\frac{A_{v}}{\mu_{v}}, E_{v}=0, I_{v}=0\right) .
\end{gathered}
$$

The local stability of the system is determined by the signs of the eigenvalues and it is further proved by linearizing to obtain its Jacobian at disease-free steady-state points so that

$$
J_{d f e}=\left(\begin{array}{ccccccccc}
-J_{1} & 0 & 0 & 0 & 0 & \tau & 0 & 0 & 0 \\
\delta & -J_{2} & 0 & 0 & 0 & 0 & 0 & 0 & 0 \\
0 & \epsilon \sigma_{h} & -J_{3} & 0 & 0 & 0 & 0 & 0 & 0 \\
0 & 0 & \psi & -J_{4} & 0 & 0 & 0 & 0 & 0 \\
0 & 0 & 0 & 0 & -J_{5} & 0 & 0 & 0 & 0 \\
0 & 0 & \phi_{1} & \phi_{2} & v \phi+\phi_{3} & -J_{6} & 0 & 0 & 0 \\
0 & 0 & 0 & 0 & 0 & 0 & -J_{7} & 0 & 0 \\
0 & 0 & 0 & 0 & 0 & 0 & 0 & -J_{8} & 0 \\
0 & 0 & 0 & 0 & 0 & 0 & 0 & \sigma_{v} & -J_{9}
\end{array}\right) .
$$

The eigenvalues of the system above satisfy $-J_{9}<0,-J_{8}<0,-J_{7}<0,-J_{5}<$ 
0 , and the remaining characteristics polynomial is given as

$$
\begin{array}{r}
\lambda^{5}-\left(J_{1}+J_{2}+J_{3}+J_{4}+J_{6}\right) \lambda^{4} \\
+\left(J_{1} J_{2}+J_{1} J_{3}+J_{1} J_{4}+J_{1} J_{6}+J_{2} J_{3}+J_{2} J_{4}\right. \\
\left.+J_{2} J_{6}+J_{3} J_{6}+J_{4} J_{6}\right) \lambda^{3}-\left(J_{1} J_{2} J_{3}+J_{1} J_{2} J_{4}+J_{1} J_{2} J_{6}+J_{1} J_{3} J_{4}\right. \\
\left.+J_{1} J_{3} J_{6}+J_{1} J_{4} J_{6}+J_{2} J_{3} J_{4}+J_{2} J_{3} J_{6}+J_{2} J_{4} J_{6}+J_{3} J_{4} J_{6}\right) \lambda^{2} \\
+\left(-\delta \tau \phi_{1} \epsilon \sigma_{h}+J_{1} J_{2} J_{3} J_{4}+J_{1} J_{2} J_{3} J_{6}+J_{1} J_{2} J_{4} J_{6}\right. \\
\left.+J_{1} J_{3} J_{4} J_{6}+J_{2} J_{3} J_{4} J_{6}\right) \lambda \\
-\left(\phi_{2} \psi \epsilon \sigma_{h} \delta \tau+\phi_{1} \epsilon \sigma_{h} \delta \tau J_{4}-J_{1} J_{2} J_{3} J_{4}, J_{6}\left(1-R_{m f}\right)\right) .
\end{array}
$$

Clearly, in the trace-determinant plane, the trace of (39) is

$$
-\left(J_{9}+J_{8}+J_{7}+J_{6}+J_{5}+J_{4}+J_{3}+J_{2}+J_{1}\right)<0
$$

and the determinant of (39) is

$$
J_{9} J_{8} J_{7} J_{5}\left(-\delta \tau \phi_{1} \epsilon \sigma_{h}-\delta \tau J_{4} \phi_{1} \epsilon \sigma_{h}+J_{1}+J_{2}+J_{3}+J_{4}+J_{6}\right)>0 .
$$

Hence by the Routh-Hurwitz criteria [25], all the eigenvalues have negative real parts, which proves that the disease-free equilibrium is locally asymptotically stable when $R_{m f}<1$.

\subsection{Global stability of disease-free steady state}

Theorem 4.2. $[27,28]$ The disease-free steady state of system (13) is globally asymptotically stable whenever $R_{m f}<1$.

Proof. From the theorem of Castillo - Chavez et al. [27], given that

$$
\begin{aligned}
\dot{X} & =F(X, Z), \\
\dot{Z} & =G(X, Z),
\end{aligned}
$$

where $X=\left(S_{h}, S_{2}, T, S_{v}\right)$ represents the total numbers of compartments without infections, such that $X \in \Re^{+4}$ and $Z=\left(I_{m}, I_{f}, I_{m f}, I_{v}, E_{v}\right)$ represents the total number of compartments with infections. The disease-free equilibrium solutions is denoted as $E^{0}=\left(X^{*}, Z^{*}\right)=\left(X^{*}, 0\right)$, where $X^{*}=\left(\frac{A_{h}}{\mu_{h}}, \frac{\pi A_{h}}{\mu_{h}}, \frac{A_{v}}{\mu_{v}}\right)$. The following conditions hold:

i $\dot{X}=F(X, 0), X^{*}$ is globally asymptotically stable, 
ii $G(X, Z)=D_{Z}\left(X^{*}, 0\right) Z-\hat{G}(X, Z) \geq 0$ for all $(\mathrm{X}, \mathrm{Z}) \in \Delta$, where $\Delta$ is a region where the model system (13) makes an epidemiological sense,

where $D_{Z} G\left(X^{*}, 0\right)$ denotes the Metzler matrix with nonnegative off-diagonal elements.

Therefore,

$$
F(X, 0)=\left(\begin{array}{c}
\pi A_{h}-\left(\mu_{h}+\delta\right) S_{h}+\tau T \\
(1-\pi) A_{h}+\delta S_{h}-\left(\epsilon \sigma_{h}+\gamma+\mu_{h}\right) S_{2} \\
-\left(\mu_{h}+\tau\right) T \\
\lambda_{v}-\mu_{v} S_{v}
\end{array}\right)
$$

and

$$
G(X, Z)=\left(\begin{array}{c}
-\theta I_{m} K_{f}-\left(\psi+\phi_{1}+\mu_{h}\right) I_{m} \\
\psi I_{m}-\rho K_{m} I_{f}-\left(\gamma+\phi_{2}+\mu_{h}\right) I_{f} \\
\theta K_{f} I_{m}+\rho K_{m} I_{f}-\left(\phi_{3}+v \phi+\eta \gamma+\mu_{h}\right) I_{m f} \\
-\left(\sigma_{v}+\mu_{v}\right) E_{v} \\
-\mu_{v} I_{v}
\end{array}\right) .
$$

In the absence of disease, it is clear that $G(X, 0)=0$. Also

$$
D_{Z} G\left(X^{*}, 0\right)=\left(\begin{array}{ccccc}
-m_{1} & 0 & 0 & 0 & 0 \\
\psi & -m_{2} & 0 & 0 & 0 \\
0 & 0 & -m_{3} & 0 & 0 \\
0 & 0 & 0 & -m_{4} & 0 \\
0 & 0 & 0 & \sigma_{v} & -m_{5}
\end{array}\right)
$$

where $m_{1}=\left(\psi+\phi_{1}+\mu_{h}\right), m_{2}=\left(\gamma+\phi_{2}+\mu_{h}\right), m_{3}=\left(\phi_{3}+v \phi+\eta \gamma+\mu_{h}\right)$, $m_{4}=\left(\sigma_{v}+\mu_{v}\right), m_{5}=\mu_{v}$.

so that

$$
\hat{G}(X, Z)=\left(\begin{array}{c}
-\theta\left(I_{m}^{*}-I_{m}\right) K_{f}-m_{1}\left(I_{m}^{*}-I_{m}\right) \\
\psi\left(I_{m}^{*}-I_{m}^{*}\right)-\rho K_{m}\left(I_{f}^{*}-I_{f}\right)-m_{2}\left(I_{f}^{*}-I_{f}\right) \\
\theta K_{f}\left(I_{m}^{*}-I_{m}\right)+\rho K_{m}\left(I_{f}^{*}-I_{f}\right)-m_{4}\left(I_{m f}^{*}-I_{m f}\right) \\
-m_{4}\left(E_{v}^{*}-E_{v}\right) \\
-m_{5}\left(I_{v}^{*}-I_{v}\right)
\end{array}\right) .
$$

From the first condition in the stated proof above, $S_{h}(t)=\frac{A_{h}}{\mu_{h}}+\left(S_{h}(0)-\right.$ $\left.\frac{A_{h}}{\mu_{h}}\right) e^{-\mu_{h} t} \rightarrow \frac{A_{h}}{\mu_{h}}$ as $t \rightarrow \infty, S_{2}(t)=\frac{\pi A_{h}}{\mu_{h}}+\left(S_{2}(0)-\frac{\pi A_{h}}{\mu_{h}}\right) e^{-\mu_{h} t} \rightarrow \frac{\pi A_{h}}{\mu_{h}}$ 
as $t \rightarrow \infty$ and $S_{v}(t)=\frac{A_{v}}{\mu_{v}}+\left(S_{v}(0)-\frac{A_{v}}{\mu_{v}}\right) e^{-\mu_{v} t} \rightarrow \frac{A_{v}}{\mu_{v}}$ as $t \rightarrow \infty$. Since $X^{*}=\left(\frac{A_{h}}{\mu_{h}}, \frac{\pi A_{h}}{\mu_{h}}, \frac{A_{v}}{\mu_{v}}\right), X^{*}$ is globally asymptotically stable.

Also, if $I_{m}^{*}=I_{m}, I_{f}^{*}=I_{f}, I_{m f}^{*}=I_{m f}, E_{v}^{*}=E_{v}, I_{v}^{*}=I_{v}$, then the disease-free steady-state points denoted as $I_{m}^{*}, I_{f}^{*}, I_{m f}^{*}, E_{v}^{*}, I_{v}^{*}$, are all zeros. Then, $\hat{G}(X, Z) \geq$ 0 . Therefore, from the second condition in the stated theorem that $G(X, Z)=$ $D_{Z}\left(X^{*}, 0\right) Z-\hat{G}(X, Z) \geq 0$, the disease-free steady state $E^{0}=\left(X^{*}, 0\right)$ is globally asymptotically stable.

\subsection{Local stability of endemic steady state}

The possibility of the existence of a backward bifurcation is investigated to establish the local stability of the endemic equilibrium of the system. If $R_{m f}<1$, there is the possibility of a backward bifurcation. Also, $R_{m f}>1$, the disease persists and become endemic and $R_{m f}=1$ is the bifurcation point where a forward bifurcation occurs.

Theorem 4.3. $[27,28]$ If $R_{m f}=1$, then $R_{m f}=1$ is the bifurcation value where the stability of the system changes.

Proof. The center manifold theorem is employed, where the following change in notations of variables was made. Let $S_{h}=x_{1}, S_{2}=x_{2}, I_{m}=x_{3}, I_{f}=x_{4}, I_{m f}=$ $x_{5}, T=x_{6}, S_{v}=x_{7}, E_{v}=x_{8}, I_{v}=x_{9}$, and $\beta k=\beta k^{*}$ denote the bifurcation value and $R_{m f}=1$ as the bifurcation point. That is,

$$
1=\frac{\tau \delta\left(\psi \phi_{2}-J_{4} \phi_{3}\right) \epsilon \sigma_{h}+J_{1} J_{2} J_{3} J_{4} J_{5} J_{6}}{\epsilon \sigma_{h}\left(J_{6} J_{1}\left(\beta k^{*} \psi-\beta J_{4}\right)+\tau \delta\left(\psi \theta \beta k^{*}-\theta \beta J_{4}\right)\right)},
$$

where $\beta k^{*}=\frac{1}{R_{m f}}$. The model of system (13) is redefined as

$$
\begin{aligned}
& \dot{x_{1}}=\pi A_{h}-K_{m a} x_{1}-K_{f} x_{1}-\delta x_{1}+\tau x_{6}, \\
& \dot{x_{2}}=(1-\pi) A_{h}+\delta x_{1}+K_{m a} x_{1}+K_{f} x_{1}-\left(\epsilon \sigma_{h}+\gamma+\mu_{h}\right) x_{2}, \\
& \dot{x_{3}}=\epsilon \sigma_{h} x_{2}-\theta K_{f} x_{3}-\left(\psi+\phi_{1}+\mu_{h}\right) x_{3}, \\
& \dot{x_{4}}=\psi x_{3}-\rho K_{m} a x_{4}-\left(\gamma+\phi_{2}+\mu_{h}\right) x_{4}, \\
& \dot{x_{5}}=\theta K_{f} x_{3}+\rho K_{m a} x_{4}-\left(\phi_{3}+v \phi+\eta \gamma+\mu_{h}\right) x_{5}, \\
& \dot{x_{6}}=v \phi x_{5}+\left(\phi_{1} x_{3}+\phi_{2} x_{4}+\phi_{3} x_{5}\right)-\mu_{h} x_{6}-\tau x_{6}, \\
& \dot{x_{7}}=A_{v}-K_{v} x_{7}-\mu_{v} x_{7}, \\
& \dot{x_{8}}=K_{v} x_{7}-\left(\sigma_{v}+\mu_{v}\right) x_{8}, \\
& \dot{x_{9}}=\sigma_{v} x_{8}-\mu_{v} x_{9} .
\end{aligned}
$$


The Jacobian of the system (13) is given by

$$
\left(\begin{array}{ccccccccc}
-J_{1} & 0 & 0 & 0 & 0 & \tau & 0 & 0 & 0 \\
\delta & -J_{2} & 0 & 0 & 0 & 0 & 0 & 0 & 0 \\
0 & \epsilon \sigma_{h} & -J_{3} & 0 & 0 & 0 & 0 & 0 & 0 \\
0 & 0 & \psi & -J_{4} & 0 & 0 & 0 & 0 & 0 \\
0 & 0 & 0 & 0 & -J_{5} & 0 & 0 & 0 & 0 \\
0 & 0 & \phi_{1} & \phi_{2} & v \phi+\phi_{3} & -J_{6} & 0 & 0 & 0 \\
0 & 0 & 0 & 0 & 0 & 0 & -J_{7} & 0 & 0 \\
0 & 0 & 0 & 0 & 0 & 0 & 0 & -J_{8} & 0 \\
0 & 0 & 0 & 0 & 0 & 0 & 0 & \sigma_{v} & -J_{9}
\end{array}\right),
$$

where $J_{1}=\left(\mu_{h}+\delta\right), J_{2}=\left(\epsilon \sigma_{h}+\gamma+\mu_{h}\right), J_{3}=\left(\psi+\phi_{1}+\mu_{h}\right), J_{4}=\left(\gamma+\phi_{2}+\mu_{h}\right)$, $J_{5}=\left(\phi_{3}+v \phi+n \gamma+\mu_{h}\right), J_{6}=\left(\mu_{h}+\tau\right), J_{7}=\mu_{v}, J_{8}=\left(\sigma_{v}+\mu_{v}\right), J_{9}=\mu_{v}$. We now proceed to calculate the left and right eigenvectors associated with (50). Let $w=\left(w_{1}, w_{2}, w_{3}, w_{4}, w_{5}, w_{6}, w_{7}, w_{8}, w_{9}\right)$ be the right eigenvector associated with the matrix (50), then, we obtain

$$
\begin{array}{r}
w_{1}=\frac{\tau}{(\mu+\delta)} w_{6}, w_{2}=\frac{\left(\psi+\phi_{1}+\mu_{h}\right)}{\epsilon \sigma_{h}} w_{3}, \\
w_{3}=\frac{\left(\gamma+\phi_{2}+\mu_{h}\right)}{\psi} w_{4}, \quad w_{4}=w_{4}>0, \\
w_{5}=w_{5}>0, \quad w_{6}=\frac{\phi_{1}}{\mu_{h}+\tau} w_{4}, \\
w_{7}=0, \quad w_{8}=w_{8}>0, \quad w_{9}=w_{9}>0 .
\end{array}
$$

Also, for $v=\left(v_{1}, v_{2}, v_{3}, v_{4}, v_{5}, v_{6}, v_{7}, v_{8}, v_{9}\right)^{T}$, we obtain

$$
\begin{array}{r}
v_{1}=\frac{\delta}{J_{1}} v_{2}, v_{2}=\frac{\epsilon \sigma_{h} v_{3}}{J_{2}}, v_{3}=v_{3}>0, \quad v_{4}=\frac{\phi_{2}}{J_{4}} v_{6}, \\
v_{5}=\frac{v \phi+\phi_{3}}{J_{5}} v_{6}, \quad v_{6}=\frac{J_{3} v_{3}}{J_{4}\left(\psi \phi_{2}+\phi_{1}\right)}, v_{7}=0, v_{8}=0, \quad v_{9}=0 .
\end{array}
$$

Next, the following theorem is used.

Theorem 4.4. $[19,28]$ Consider the general system of differential equations with a parameter $\mu$ such that

$$
\frac{d x}{d t}=f(x, \mu), f: \Re^{n} \times \Re^{+} \rightarrow \Re^{+},
$$


and $f \in C^{2}\left(\Re^{n} \times \Re\right)$, and 0 is an equilibrium point of the system for all $\mu$ and

$H 1 B=D_{x} f(0,0)=\frac{\partial f_{i}(0,0)}{\partial x_{j}}$ is the linearization matrix of the system around the equilibrium 0 with $\theta$ evaluated at 0 .

H2 Zero is a simple eigenvalue of $B$ and all other eigenvalues of $B$ have negative real parts.

$H 3$ The matrix $B$ has a right eigenvector $w$ and left eigenvector $v$ corresponding to the zero eigenvalue.

If $f_{k}$ is the $k^{\text {th }}$ component of $f$, then

$i \quad a_{o}=\sum_{k, i, j=1}^{n} w_{k} v_{i} v_{j} \frac{\partial^{2} f_{k}(0,0)}{\partial x_{i} \partial x_{j}}$,

ii $b_{o}=\sum_{k, i, j=1}^{n} w_{k} v_{i} v_{j} \frac{\partial^{2} f_{k}(0,0)}{\partial x_{i} \partial \beta k^{*}}$,

the local dynamics of the system (13) at the equilibrium solutions is determined by the signs of $a_{o}$ and $b_{o}$, especially, if $a_{o}>0$ and $b_{o}>0$, then $a$ subcritical (or backward) bifurcation occurs at $\mu=0$.

iii $a_{o}>0, b_{o}>0$ : when $\mu<0$ with $|\mu|<<1,0$ is locally asymptotically stable and there exists a positive unstable equilibrium; when $0<\mu<<$ 1,0 is unstable and there exists a negative and locally asymptotically stable equilibrium.

iv $a_{o}<0, b_{o}<0$ : when $\mu<0$ with $|\mu|<<1,0$ is unstable; when $0<\mu<<$ 1,0 is unstable and there exists a positive and unstable equilibrium.

$v a_{o}>0, b_{o}<0$ : when $\mu<0$ with $|\mu|<<1,0$ is stable and there exists $a$ locally asymptotically stable negative equilibrium; when $0<\mu<<1,0$ is unstable and there exists a positive and unstable equilibrium.

vi $a_{o}<0, b_{o}>0$ : when $\mu<0$, if $\mu$ changes from negative to positive and 0 changes from stable to unstable, there exists a positive and unstable equilibrium. Also, a negative unstable equilibrium becomes positive and locally asymptotically stable if $a_{o}>0$ and $b_{o}>0$, then a backward bifurcation occurs at $\mu=0$. 


\section{Computations of $a_{o}$ and $b_{o}$}

To compute for $a_{o}$ for the transformed system of (50), the associated nonzero partial derivatives of $f$ (evaluated at the disease-free steady state) are given by

$$
\begin{aligned}
\dot{x_{1}} & =f_{1}\left(x_{1}, x_{2}, \ldots, x_{9}\right)=\pi A_{h}-K_{m a} x_{1}-K_{f} x_{1}-\delta x_{1}+\tau x_{6}, \\
\dot{x_{2}} & =f_{2}\left(x_{1}, x_{2}, \ldots, x_{9}\right)=(1-\pi) A_{h}+\delta x_{1}+K_{m a} x_{1}+K_{f} x_{1} \\
& -\left(\epsilon \sigma_{h}+\gamma+\mu_{h}\right) x_{2}, \\
\dot{x_{3}} & =f_{3}\left(x_{1}, x_{2}, \ldots, x_{9}\right)=\epsilon \sigma_{h} x_{2}-\theta K_{f} x_{3}-\left(\psi+\phi_{1}+\mu_{h}\right) x_{3}, \\
\dot{x_{4}} & =f_{4}\left(x_{1}, x_{2}, \ldots, x_{9}\right)=\psi x_{3}-\rho K_{m} a x_{4}-\left(\gamma+\phi_{2}+\mu_{h}\right) x_{4}, \\
\dot{x_{5}} & =f_{5}\left(x_{1}, x_{2}, \ldots, x_{9}\right)=\theta K_{f} x_{3}+\rho K_{m a} x_{4}-\left(\phi_{3}+v \phi+\eta \gamma+\mu_{h}\right) x_{5}, \\
\dot{x_{6}} & =f_{6}\left(x_{1}, x_{2}, \ldots, x_{9}\right)=v \phi x_{5}+\left(\phi_{1} x_{3}+\phi_{2} x_{4}+\phi_{3} x_{5}\right)-\mu_{h} x_{6}-\tau x_{6}, \\
\dot{x_{7}} & =f_{7}\left(x_{1}, x_{2}, \ldots, x_{9}\right)=A_{v}-K_{v} x_{7}-\mu_{v} x_{7}, \\
\dot{x_{8}} & =f_{8}\left(x_{1}, x_{2}, \ldots, x_{9}\right)=K_{v} x_{7}-\left(\sigma_{v}+\mu_{v}\right) x_{8}, \\
\dot{x_{9}} & =f_{9}\left(x_{1}, x_{2}, \ldots, x_{9}\right)=\sigma_{v} x_{8}-\mu_{v} x_{9},
\end{aligned}
$$

where

$$
\begin{gathered}
\frac{\partial^{2} f_{1}}{\partial x_{1} \partial x_{9}}=-\alpha a, \frac{\partial^{2} f_{1}}{\partial x_{1} \partial x_{3}}=-\beta=\frac{\partial^{2} f_{1}}{\partial x_{5} \partial x_{1}}, \frac{\partial^{2} f_{1}}{\partial x_{4} \partial x_{1}}=-\beta k \\
\frac{\partial^{2} f_{2}}{\partial x_{4} \partial x_{1}}=\beta k, \frac{\partial^{2} f_{2}}{\partial x_{1} \partial x_{9}}=\alpha a, \frac{\partial^{2} f_{2}}{\partial x_{1} \partial x_{3}}=\beta=\frac{\partial^{2} f_{2}}{\partial x_{5} \partial x_{1}}, \quad \frac{\partial^{2} f_{4}}{\partial x_{9} x_{4}}=-\rho \alpha a \\
\frac{\partial^{2} f_{5}}{\partial x_{3}^{2}}=\theta \beta=\frac{\partial^{2} f_{5}}{\partial x_{5} x_{3}}, \frac{\partial^{2} f_{5}}{\partial x_{4} \partial x_{3}}=\theta \beta k, \frac{\partial^{2} f_{5}}{\partial x_{5} x_{4}}=\rho \alpha a \\
\frac{\partial^{2} f_{7}}{\partial x_{3} x_{7}}=-\alpha b, \frac{\partial^{2} f_{7}}{\partial x_{5} x_{7}}=-\alpha b \delta, \frac{\partial^{2} f_{8}}{\partial x_{3} x_{7}}=\alpha b, \frac{\partial^{2} f_{8}}{\partial x_{5} x_{2}}=\alpha b \delta
\end{gathered}
$$

A direct calculation from the expression above yield

$$
\begin{aligned}
a_{o}= & v_{1} \sum_{i, j=1}^{9} w_{i} w_{j} \frac{\partial^{2} f_{1}}{\partial x_{i} \partial x_{j}}+v_{2} \sum_{i, j=1}^{9} w_{i} w_{j} \frac{\partial^{2} f_{2}}{\partial x_{i} \partial x_{j}} \\
& +v_{3} \sum_{i, j=1}^{9} w_{i} w_{j} \frac{\partial^{2} f_{3}}{\partial x_{i} \partial x_{j}}+v_{4} \sum_{i, j=1}^{9} w_{i} w_{j} \frac{\partial^{2} f_{4}}{\partial x_{i} \partial x_{j}}
\end{aligned}
$$




$$
\begin{gathered}
+v_{5} \sum_{i, j=1}^{9} w_{i} w_{j} \frac{\partial^{2} f_{5}}{\partial x_{i} \partial x_{j}}+v_{6} \sum_{i, j=1}^{9} w_{i} w_{j} \frac{\partial^{2} f_{6}}{\partial x_{i} \partial x_{j}} \\
+v_{7} \sum_{i, j=1}^{9} w_{i} w_{j} \frac{\partial^{2} f_{7}}{\partial x_{i} \partial x_{j}}+v_{8} \sum_{i, j=1}^{9} w_{i} w_{j} \frac{\partial^{2} f_{8}}{\partial x_{i} \partial x_{j}} \\
+v_{9} \sum_{i, j=1}^{9} w_{i} w_{j} \frac{\partial^{2} f_{9}}{\partial x_{i} \partial x_{j}} .
\end{gathered}
$$

Since $v_{3}=v_{7}=v_{8}=v_{9}=0$ in (52), after some re-arrangement and simplification, (54) and (55) yield

$$
\begin{gathered}
a_{o}=v_{1}\left(-\alpha a w_{1} w_{9}-\beta\left(w_{1} w_{3}+w_{1} w_{5}\right)-\beta k\left(w_{1} w_{4}\right)\right. \\
+v_{2}\left(\alpha a w_{1} w_{9}+\beta\left(w_{1} w_{3}+w_{1} w_{5}\right)-\beta\left(w_{1} w_{3}+w_{1} w_{5}\right)+\beta k\left(w_{1} w_{4}\right)-v_{4}\left(\rho \alpha a\left(w_{4} w_{9}\right)\right)\right) \\
+v_{5}\left(\theta \beta\left(w_{3}^{2}+w_{1} w_{5}\right)+\theta \beta k\left(w_{3} w_{4}\right)+\rho \alpha a\left(w_{4} w_{9}\right)\right)>0
\end{gathered}
$$

To compute for $b_{o}$ for the transformed system of (50), the associated nonzero partial derivatives of $f$ (evaluated at the disease-free steady state) are given by

$$
\frac{\partial^{2} f_{2}}{\partial x_{4} \partial \beta k^{*}}=\beta, \frac{\partial^{2} f_{5}}{\partial x_{3} \partial \beta k^{*}}=\theta \beta,
$$

so that

$$
\begin{gathered}
b_{o}=v_{2} w_{4} \frac{\partial^{2} f_{2}}{\partial x_{4} \partial \beta k^{*}}+v_{5} w_{3} \frac{\partial^{2} f_{5}}{\partial x_{3} \partial \beta k^{*}}, \\
b_{o}=\beta\left(v_{2} w_{4}\right)+\theta \beta\left(v_{5} w_{3}\right)>0 .
\end{gathered}
$$

Since $a_{o}>0, b_{o}>0$, then the model system (13) will undergo a subcritical bifurcation, otherwise it will exhibit a forward bifurcation. Hence the endemic equilibrium is locally asymptotically stable. 


\section{Numerical simulations}

Table 1. Variables in Model (13) and Their Meanings

\begin{tabular}{|c|c|c|c|}
\hline Variable & Descriptions & Values & Source \\
\hline $\mathrm{S}_{h}(0)$ & Antenatal compliant susceptible pregnant women & 50 & Assumed \\
$\mathrm{S}_{2}(0)$ & Antenatal noncompliant susceptible pregnant women & 30 & Assumed \\
$\mathrm{I}_{m}(0)$ & Malaria infected pregnant women & 10 & Assumed \\
$\mathrm{I}_{f}(0)$ & Filariasis infected pregnant women & 15 & Assumed \\
$\mathrm{I}_{m f}(0)$ & Malaria - filariasis infected pregnant women & 20 & Assumed \\
$\mathrm{T}(0)$ & Treatment of malaria - filariasis infected pregnant women & 10 & Assumed \\
$\mathrm{S}_{v}(0)$ & Susceptible mosquitos & 25 & Assumed \\
$\mathrm{E}_{v}(0)$ & Exposed mosquitos & 20 & Assumed \\
$\mathrm{I}_{v}(0)$ & Infected mosquitos & 10 & Assumed \\
\hline
\end{tabular}

Table 2. Parameter Values and Descriptions

\begin{tabular}{|c|c|c|c|}
\hline Parameters & Symbols & Values & Source \\
\hline $\begin{array}{c}\text { Recruitment rate of } \\
\text { susceptible pregnant women }\end{array}$ & $\pi A_{h}$ & $4.21 \times 10^{-5}$ & [19] \\
\hline Per capita birth rate of mosquitos & $\lambda_{v}$ & 1000 & Varies \\
\hline Per capita natural mortality rate of pregnant women & $\mu_{h}$ & 0.00004 & Assumed \\
\hline Per capita natural mortality rate of mosquitos & $\mu_{v}$ & 0.05 & {$[16]$} \\
\hline Proportion of noncompliant susceptibles & $(1-\pi) A_{h}$ & $3 \times 10^{-5}$ & Varies \\
\hline $\begin{array}{l}\text { Progression rate from compliant } \\
\text { to noncompliant class }\end{array}$ & $\delta_{1}$ & 0.12 & Assumed \\
\hline $\begin{array}{c}\text { Rate of loss of immunity } \\
\text { to both malaria and filarisis }\end{array}$ & $\tau$ & 0.0006 & Assumed \\
\hline Rate of malaria symptoms in pregnant women & $\sigma_{h}$ & 0.8331 & {$[16,23]$} \\
\hline Modification parameter & $\epsilon$ & 0.7 & Assumed \\
\hline Modification parameter & $\theta$ & 1.2 & Assumed \\
\hline Modification parameter & $\rho$ & 1.4 & Assumed \\
\hline Modification parameter & $\delta$ & 0.00211 & Assumed \\
\hline Modification parameter & $k$ & 0.011 & Assumed \\
\hline Modification parameter & $\eta$ & 0.003 & Assumed \\
\hline Modification parameter & $v$ & 0.0036 & Assumed \\
\hline Disease induced death rate of the codisease & $\gamma$ & 0.00231 & [19] \\
\hline Progression rate of malaria leading to filariasis symptoms & $\psi$ & 1.0 & [23] \\
\hline Biting rate of mosquitos & $\alpha$ & 0.2 & [19] \\
\hline Transmission probability of malaria in mosquitos & $b$ & 0.09 & Varies \\
\hline Transmission probability of malaria in humans & $a$ & 0.8333 & {$[16]$} \\
\hline Rate of mosquito bite leading to malaria infection & $\beta I_{m}$ & 0.0183 & {$[16,20]$} \\
\hline Rate of mosquito bite leading to filariasis infection & $\beta I_{f}$ & $3.6 \times 10^{-5}$ & [23] \\
\hline Rate of mosquito bite leading to codisease infection & $\beta I_{m f}$ & $2.7 \times 10^{5}$ & [23] \\
\hline Treatment rate of malaria & $\phi_{1}$ & 0.00341 & Assumed \\
\hline Treatment rate of filariasis & $\phi_{2}$ & 0.00061 & Assumed \\
\hline Treatment rate of malaria - filariasis & $\phi_{3}$ & 0.00072 & Assumed \\
\hline Transmission rate between & $K_{v}$ & 0.502 & {$[23]$} \\
\hline susceptible and infected mosquitos & & & \\
\hline Natural death rate of mosquitos & $\mu_{v}$ & 0.1429 & {$[16,23]$} \\
\hline $\begin{array}{l}\text { Progression rate of exposed } \\
\text { to infected mosquitos }\end{array}$ & $\sigma$ & 0.0112 & Assumed \\
\hline
\end{tabular}

Figure 1 shows the profile of the pregnant women treated from malaria - filariasis coinfection by varying $\phi_{3}$ between $0.00021-0.00072$. The decline in the graph shows that use of drugs, e.g., ACT and Mectizan, will bring about the drastic 
reduction of the codisease in their system.

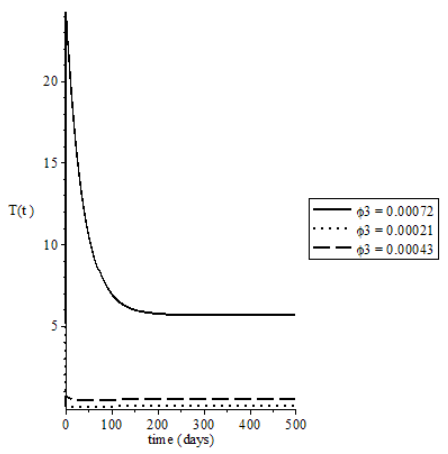

Figure 1. Graph of Disease Profile of $T$ against Time $(t)$

Figure 2 presents the disease profile of pregnant women infected with malaria filariasis. The rise in the graph shows that, in the absence of medical interventions, there will be a full blown epidemic leading to mortality and morbidity.

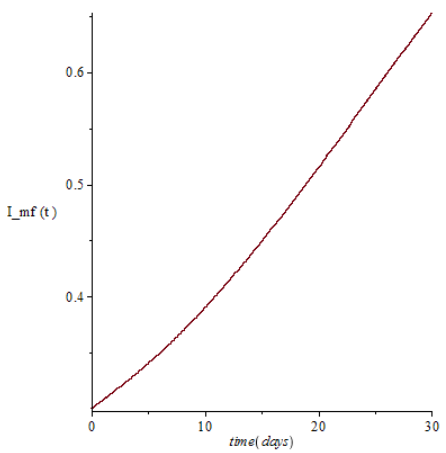

Figure 2. Graph of $I_{m f}$ against Time $(t)$

Figure 3 shows the disease profile of the susceptible pregnant women non compliant to medical antenatal program. The rise in the graph depicts the group having a high tendency of contacting the malaria - filariasis coinfection. 


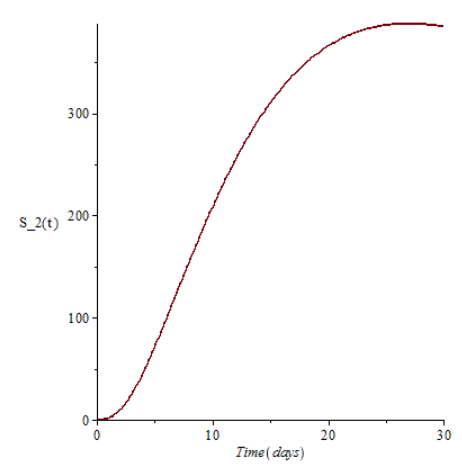

Figure 3. Graph of $S_{2}$ against Time $(t)$

Figure 4 presents the variation of the loss of immunity rate $\tau$ between $0.0002-$ 0.0006. The decline shows that after appropriate treatment, they lose immunity overtime, thereby making them susceptible to the coinfection.

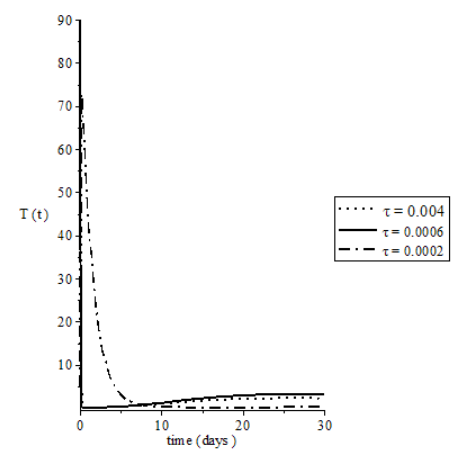

Figure 4. Graph of $T$ against Time $(t)$

Figure 5 illustrates the disease profile's number of deaths of Pregnant women infected with malaria and filariasis in the absence of medical interventions, varying the disease induced death rate $\gamma$ between $0.00176-0.00231$. 


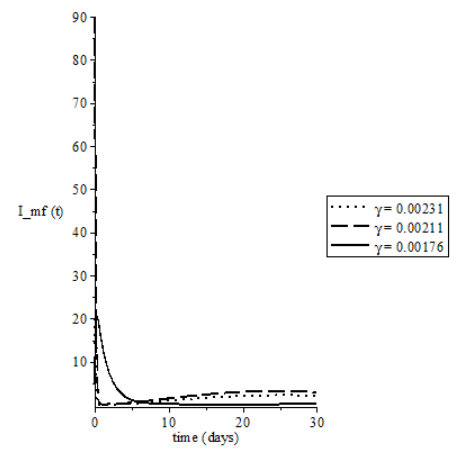

Figure 5. Graph of $I_{m f}$ against Time $(t)$

Figure 6 shows the $S_{h}$ group of pregnant women who are susceptible to malaria - filariasis coinfections but are still compliant to antenatal medical program. The gradual rise and decline on the graph shows that the coinfections will be minimized overtime with their compliance to antenatal program.

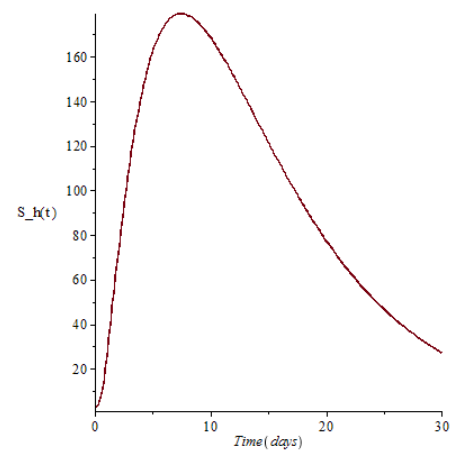

Figure 6. Graph of $S_{h}$ against Time $(t)$

\section{Conclusion and recommendations}

A deterministic model describing the transmission of malaria - filariasis coinfections in susceptible host of pregnant women population is considered. A domain where the model is well posed and realistic in an epidemic sense is presented. Also, the basic reproduction number of malaria - filariasis coinfections $\left(R_{m f}\right)$ is obtained and the local and global behavior is analyzed. It was investigated that if $R_{m f}<1$, malaria - filariasis coinfections vanish out of the population, and if $R_{m f}>1$, the coinfections persist and become endemic. However, variations 
in the values of state variables and parameters involved in the model formulation bring about the need for medical intervention strategies like treatment, to curtail the spread of the coinfectious disease. This work can be further extended by considering the impact of climatic factors, optimal controls, cost effective strategies.

Acknowledgments. I dedicate this research article to my parents, $\mathrm{Mr}$ and Mrs M. A. Ogunmiloro, and to my wife and children namely Damilola, Ayobami and Emmanuel. I acknowledge the assistance of my family, friends and academic colleagues at the Ekiti State University and University of Ilorin, Nigeria. I say thank you all.

\section{Bibliography}

[1] World Health Organization (WHO), Fact Sheet on Malaria, https://www . who. int/malaria/media/world-malaria-day-2018/en/.

[2] World Health Organization (WHO), Fact Sheet on Filariasis, https : //www . who. int/lymphatic_filariasis/en/-2018.

[3] S. O. Nzeako, O. H. Okunnuga, F. Nduka and E. C. Oluchi, Lymphatic filariasis and malaria amongst residents of Port-Harcourt metropolis, Intl. J. Appl. Sci. Res. Rev. 3(6) (2016), 1-11, DOI:10.21767/2349-7238.100051.

[4] G. Chandrakala and M. Zulfeen, Filariasis in pregnancy: Prevalent, yet less known global health burden, Journal of Basic and Clinical Reproductive Sciences 5(2) (2016), 107-109.

[5] Neglected Tropical Disease Network, Nigeria Launches Africa's First Nation wide Malaria and Lymphatic Filariasis Co-Implementation Plan, in: Federal Ministry of Health Abuja, http: //www.ntd-ngdonetwork .org/news-events / news/nigeria-launches-africa2014.

[6] M. Bal, M. Ranjit, K. G. Achary and A. K. Satapathy, Maternal filarial infection influences the development of regulatory $t$ cells in children from infancy to early childhood, PLoS. Negl. Trop. Dis. 10(11) (2016), e0005144, DOI:10.1371/journal.pntd.0005144.

[7] Daily Trust, Nigeria: 70 Million Nigerians Need Treatment against Elephantiasis, in: https://allafrica.com/stories/201203280692.html, Health in Nigeria, 27 March 2012.

[8] A. G. Abdullahi, I. A. Alaku and S. B. Hudu, Epidemiology of malaria and filariasis, prevention and control program in rural Nassarawa State, Nigeria, Journal of Biology, Agriculture and Health Care 5(2) (2015), 55-57.

[9] A. A. Adegnika, M. Ramharter, S. T. Agnandji, U. Ateba Ngoa, S. Issifou, M. Yazdanbahksh and P. G. Kremsner, Epidemiology of parasitic coinfection during pregnancy in Lambarene, Gabon, Tropical Medicine and International Health 15(10) (2010), 1204 - 1209. 
[10] H. W. Hethcote, A thousand and one epidemic models, in: Frontiers in Theoretical Biology, S. A. Levin, ed., Lecture Notes in Biomath. Vol. 100, Springer-Verlag, Berlin, 1994, pp. 504-515.

[11] R. M. Anderson and R. M. May, Infectious Disease of Humans: Dynamics and Control, Oxford University Press, London, UK, 1999.

[12] C. P. Bhunu and S. Mushayabasa, Transmission dynamics of lymphatic filariasis: A mathematical approach, ISRN Biomathematics 2012 (2012), Article ID:930130, 9 pages.

[13] S. O. Nzeako, Prevalence of malaria in pregnant women attending antenatal care at University of Port Harcourt, Rivers State, Nigeria, International Journal of Scientific Research in Environmental Sciences 1(10) (2013), 268 - 272.

[14] Fatmawati, H. Tasman, An optimal treatment control of TB-HIV coinfection, International Journal of Mathematics and Mathematical Sciences 2016 (2016), Article ID:8261208, 11 pages.

[15] J. Mensah, J. Dontwi and E. Bonyah, Stability analysis of zika - malaria coinfection model for malaria endemic region, Journal of Advances in Mathematics and Computer Science 26(1) (2018), Article No. JAMCS.37229, 22 pages.

[16] K. O. Okosun and O. D. Makinde, A coinfection model of malaria and cholera disease with optimal control, Math. Biosci. 258 (2014), 19-32.

[17] Z. Mukandavire, A. B. Gumel, W. Garira and J. M. Tchuenche, Mathematical analysis of a model for HIV-malaria co-infection, Mathematical Biosciences \& Engineering 6(2) (2009), 333-362.

[18] J. M. Mutua, F-B. Wang and N. K. Vaidya, Modeling malaria and typhoid fever co-infection dynamics, Math Biosci. 264 (2015), 128 - 144.

[19] E. A. Bakare and C. R. Nwozo, Mathematical analysis of malaria - schistosomiasis coinfection model, Epidemiology Research International 2016 (2016), Article ID:3854902, 19 pages.

[20] E. A. Bakare and C. R. Nwozo, Bifurcation and sensitivity analysis of malaria shistosomiasis coinfection model, Int. J. Appl. Comput. Math. 3(1) (2017), 971-1000.

[21] B. Traore, B. Sangare and S. Traore, A mathematical model of malaria transmission with structured vector population and seasonality, Journal of Applied Mathematics 2017 (2017), Article ID:6754097, 15 pages.

[22] P. Cull, Local and global stability for population models, Biological Cybernetics 54(3) (1986), 141-149.

[23] H. C. Slater, M. Gambhir, P. E. Parham and E. Michael, Modeling coinfection with malaria and lymphatic filariasis, PLOS Comput. Biol. 9(6) (2013), e1003096, 14 pages. 
[24] O. Diekmann, J. A. P. Heesterbeek and M. G. Roberts, The construction of nextgeneration matrices for compartmental epidemic models, J. R. Soc. Interface 7(47) (2010), 873-885.

[25] B. K. Sahu, M. M. Gupta and B. Subudhi, Stability analysis of nonlinear systems using Dynamic-Routh's stability criterion: A new approach, http://homepage .

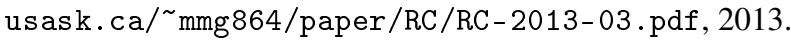

[26] J. Carr and R. G. Muncaster, Application of center manifold theory to amplitude expansions. I. Ordinary differential equations, J. Diff. Eqn. 50(2) (1983), 260 - 279.

[27] C. Castillo-Chavez, Z. Feng and W. Huang, On the Computation of $R_{0}$ and Its Role on Global Stability, IMA Vol. Math-Appl, Springer, New York, 2002.

[28] C. Castillo-Chavez and B. Song, Dynamical models of tuberculosis and their applications, Mathematics and Computers in Simulation 79(10) (2009), 3038-3054.

[29] P. M. Mwantobe, S. M. Simelane and J. M. Tchuenche, Mathematical analysis of lymphatic filariasis model with quarantine and treatment, BMC Public Health 17:265 (2017), 13 pages.

Received November 11, 2018; revised February 6, 2019, April 24, 2019; accepted May 20, 2019.

\section{Author information}

Oluwatayo M. Ogunmiloro, Department of Mathematics, Ekiti State University, Ado Ekiti, Ekiti State, P.M.B. 5363, Nigeria.

E-mail: oluwatayo.ogunmiloro@eksu.edu.ng 